\title{
Evaluation of an Interprofessional Continuing Professional Development Course on Comprehensive Diabetes Care: A Mixed- Methods Approach
}

\author{
Darrick Beckman \\ San Antonio Military Medical Center \\ Jana L. Wardian PhD \\ University of Nebraska Medical Center, jana.wardian@unmc.edu \\ Tom J. Sauerwein \\ Wilford Hall Ambulatory Surgical Center (WHASC) \\ Mark W. True \\ San Antonio Military Medical Center
}

Tell us how you used this information in this short survey.

Follow this and additional works at: https://digitalcommons.unmc.edu/com_hosp_articles

\section{Recommended Citation}

Beckman, Darrick; Wardian, Jana L. PhD; Sauerwein, Tom J.; and True, Mark W., "Evaluation of an Interprofessional Continuing Professional Development Course on Comprehensive Diabetes Care: A Mixed-Methods Approach" (2019). Journal Articles: Hospital Medicine. 10.

https://digitalcommons.unmc.edu/com_hosp_articles/10

This Article is brought to you for free and open access by the Hospital Medicine at DigitalCommons@UNMC. It has been accepted for inclusion in Journal Articles: Hospital Medicine by an authorized administrator of DigitalCommons@UNMC.For more information, please contact digitalcommons@unmc.edu. 


\title{
Evaluation of an interprofessional continuing professional development course on comprehensive diabetes care: A mixed- methods approach
}

\author{
Darrick Beckman MD, Endocrinologist $^{1}$ (D) । Jana Wardian PhD, MSW, Research Director ${ }^{2}$ (D) । \\ Tom J. Sauerwein MD, Director ${ }^{2}$ । Mark W. True MD, Interim Dean ${ }^{1}$ (D)
}

\author{
${ }^{1}$ Endocrinology Service, San Antonio Military \\ Medical Center, 3551 Roger Brooke Dr., Joint \\ Base San Antonio, Fort Sam Houston, TX \\ 78253, USA \\ ${ }^{2}$ Diabetes Center of Excellence (DCOE), \\ Wilford Hall Ambulatory Surgical Center \\ (WHASC), 1100 Wilford Hall Loop, Joint Base \\ San Antonio-Lackland, TX 78236, USA \\ Correspondence \\ Darrick Beckman, MD, Endocrinology Service, \\ San Antonio Military Medical Center, 3551 \\ Roger Brooke Dr., Joint Base San Antonio, \\ Fort Sam Houston, TX 78253, USA. \\ Email: darrick.j.beckman.mil@mail.mil
}

\begin{abstract}
Rationale: Since there are only 33 endocrinologists within the Department of Defence and over 150000 beneficiaries with diabetes, most patients with diabetes will be treated by primary care providers (PCPs). Comprehensive diabetes care visits are extensive and the clinical practice guidelines (CPGs) routinely change; thus, providing current evidence-based care is difficult. Most professional development courses aim to update PCPs on CPGs but are often inadequate as they focus on only the PCPs (not the interdisciplinary team) without a plan to implement changes into practice.
\end{abstract}

Objective: To evaluate the biannual (twice yearly), 3-day, interprofessional Diabetes Champion Course (DCC) developed by the US Air Force Diabetes Center of Excellence on comprehensive diabetes care.

Methods: A mixed-methods approach was used to evaluate three iterations of the DCC course (Sept 2014-Sept 2015). Quantitatively, pre-course and post-course surveys were used to obtain impact on knowledge, skills, and intention to change clinical practice. Qualitatively, semi-structured phone interviews were conducted with participants to obtain benefits to their clinic related to attending the DCC and barriers to implementation of the CPG process improvement project.

Results: Twelve of 19 responding clinics (63\%) reported implementing all or part of their original CPG project developed at the DCC, and 17 of 19 clinics (89\%) reported improvements associated with attending the DCC. Post-course surveys, from on location participants, revealed significant improvements in knowledge $(P<0.01)$. Likewise, foot exam skills and ability to demonstrate glucose meters to patients improved. Even with high pre-course confidence, $97 \%$ of providers reported acquiring new knowledge about prescribing and titrating insulin.

Conclusion: The DCC is innovative as it employs a team-based, interprofessional, didactic, and interactive approach that is effective in improving knowledge, skills, and intention to change clinical practice, which should translate to better care for patients with diabetes.

KEYWORDS

evaluation, evidence-based medicine, medical education 


\section{1 | INTRODUCTION}

The Department of Defence (DoD) provides comprehensive care to over 150000 patients with diabetes (active duty, retirees, and dependents) in over 400 clinics worldwide. Leaders in diabetes care, endocrinologists, are limited in number (only 33 in the DoD) and in location (only 15 Military Treatment Facilities). This problem is similar in the civilian arena as the current and future status of clinical endocrinologists indicates a current shortage of 1500 endocrinologists that would expand to 2700 by the year $2025 .{ }^{1}$ In order to meet this disparity in the DoD, primary care teams must be trained to provide standard of care for patients with diabetes in spite of limited time and resources.

Clinical practice guidelines (CPGs) are recommendations that have been thoroughly reviewed and vetted by an expert panel to provide current, evidence-based guidance that are routinely updated. Keeping up with these guidelines can be daunting. Continuing medical education/continuing professional development (CPD) appears to not fully meet this demand. In a survey of primary care physicians, there was unfamiliarity with diabetes clinical protocols, which led to poor adherence to CPGs. ${ }^{2}$ A recent American College of Cardiology/American Heart Association taskforce reviewed CPG implementation and showed that strategies of audit and feedback and educational outreach visits were effective in improving process of care, clinical outcomes, and CPG adherence. ${ }^{3}$ Furthermore, Sachveda noted that CPD courses should be aimed at the specific needs of the individual on identified gaps. ${ }^{4}$

As clinical demands change, figuring out how to effectively disseminate information and keep up with ever-changing knowledge is challenging. CPD courses have been under review as to their effectiveness. A Cochrane Review in 2009 examined articles from 1999 to 2006 finding 81 trials involving more than 11000 health professionals. ${ }^{5}$ It found that the traditional model of educational meetings alone had about a $6 \%$ improvement in compliance. However, mixed interactive and didactic educational meetings were more effective than didactic or interactive meetings alone.

Most CPD courses in the medical community are solely focused on the medical provider (ie, MD/DO) and not the interdisciplinary team. Interestingly, interprofessional development, which includes all members of a primary care team from administrative staff and technicians to providers and hospital leadership, demonstrated improved team function, built trust, and increased knowledge by allowing all team members to contribute their expertise. ${ }^{6}$

Taking knowledge back to the clinical setting and implementing it is the crucial step that can appear insurmountable for many professionals. Our facility is making strides to become a High Reliability Organization (HRO), an effort that is stressed by the Institute of Medicine. One of the tenets of an HRO is "preoccupation with failure."7 This tenet emphasizes focusing on where we fail to adhere to CPGs. Another tenet is "deference to expertise." This highlights the need to pay attention to the front line staff experts that can have invaluable insight into problems. Applying this to comprehensive diabetes care in the DoD, each local clinic will have different failures that are best identified by local staff who can develop a plan to address them based upon differing local resources. Again, the first step must ensure foundational knowledge, followed by robust process improvement $(\mathrm{PI})$ as a recommended part of an HRO. ${ }^{8}$ Dovetailing into this idea, primary health centers have shown improved delivery of health care depending on duration of participation in continuous PI. ${ }^{9}$

The US Air Force Diabetes Center of Excellence developed a CPD course, the Diabetes Champion Course (DCC), which uniquely addresses the abovementioned focuses while shoring up the shortage of diabetes experts DoD-wide. The DCC was developed in 2013 as an interprofessional, educational course for medical technicians, nurses, nurse managers, disease managers, PharmDs, dietitians, hospital leadership, and providers (MD, DO, NP, PAs) to develop local champions in diabetes standards of care, a "Diabetes Champion." This biannual (twice yearly), 3-day course provides didactic and hands-on training with focus on current standards, the latest technologies, and patient flow in a team-based setting. Additionally, each team is tasked to identify local deficits through a survey prior to course attendance and then to develop a Plan of Action (POA) to address this gap. POAs are developed as the initial PI project with emphasis on following the PDCA (Plan, Do, Check, Act) cycle, hopefully being the first of many subsequent PI projects. The course could be attended on location or via video teleconference (VTC).

Outreach organizers kept track of each clinic's POA with followup assessment via an electronic survey at 30 days, 6 months, and 12 months post course; unfortunately, response rate was poor at $10 \%$ to $15 \%, 0 \%$ to $5 \%$, and $0 \%$ to $5 \%$, respectively. Normally low response rates for surveys were also likely compounded due to the transient nature of the military population and not having a designated point of contact for each clinic. This, in addition to lack of traditional evaluations (ie, pre-testing and post-testing of knowledge, attitudes, and behaviours) left a large gap in ability to assess efficacy of the course. The purpose of this paper is to describe our experience with this course in terms of measuring its efficacy to impact diabetes care practices in the primary care setting.

\section{2 | METHODOLOGY}

In our own PI project, we sought to evaluate the DCC's efficacy to the DoD through two methods: (1) qualitative semi-structured telephone interviews to understand $\mathrm{PI}$ project progress and barriers to implementation, and (2) quantitative pre- and post-course surveys to assess knowledge, attitudes, and behaviours.

\section{1 | Qualitative semi-structured telephone interview design}

In the fall of 2015 , we coordinated one-on-one semi-structured phone interviews with past DCC participants. Ten broad questions were developed to determine what the participants' roles were in the clinic, if they implemented part or all of the POA, what barriers they encountered, and if any other benefits were seen in their clinic that directly related to attending the course, among other ideas (Table 1).

Potential respondents included those participants that attended on location or via VTC, turned in a POA, and had a valid email address from the previous three courses (Course \#5, \#6, and \#7 that were in $9 / 2014,4 / 2015$, and $9 / 2015$, respectively). Individual emails were 
TABLE 1 Semi-structured interview questions

1) Did you want to come to the Diabetes Champion Course? -Please explain.

Goal: Evaluate if desire correlated to outcomes.

2) Do you feel the Plan of Action (POA) sheet utilized was useful to develop an attainable plan of action? - Please provide suggestions to improve the CPG Plan of Action sheet.

Goal: Evaluate usability of POA sheet and for ways to improve.

3) Did you agree with the POA decided by your group? If not, what would you have done differently?

Goal: Evaluate if agreement correlated with efficacy.

4) Were you/your clinic able to implement the proposed POA (or even part of it)? -Please describe what you were able to implement.

Goal: Evaluate course efficacy.

5) What obstacles/road-blocks did you encounter?

Goal: Identify common issues to discuss with future participants and address in the course.

6) Were you able to work around the road-blocks? -If so, how?

Goal: Obtain new insights and possible examples to pass on to future course participants.

7) If you/your clinic did not implement the proposed POA (or part of it), can you explain what happened?

Goal: Identify themes of poor efficacy.

8) Is there anything you would have done differently in developing your CPG plan of action if you were able to do it over again/suggestions to future groups?

Goal: Identify common themes to help guide future participants.

9) Have you seen any other benefits/changes in your clinic from you going to the Diabetes Champion Course (ie, another project/process implemented, education, awareness, etc.)?

Goal: Evaluate translation of course knowledge into clinical improvement.

10) Comments: -Please feel free to make any other comments about the POA or about the course itself.

sent to all potential respondents in two rounds, the second round was sent 2 weeks later. The email was a template email describing what information we were seeking to gain via a telephone interview with the list of questions and the POA developed at the DCC attached. In the email, we asked for available times and a contact number in order to conduct a 10 to 15-minute phone interview to review the attached questions as well as anything else pertinent they would like to discuss. We confirmed phone interview appointments with respondents via email. The second email was sent out to participants from nonresponsive clinics; thus, if there was a representation from the clinic in the first round, that was considered sufficient.

Semi-structured phone interviews were conducted by a single interviewer (DB), guided by the 10 questions previously sent to participants. However, prompts were used to enable respondents to elaborate on areas of interest as time allowed. Phone calls lasted from 10 to 30 minutes, most lasting between 15 and 20 minutes. Notes were taken on the conversation in bulleted summaries. Broad POA categories were initially defined by the interviewer after review of all responses. This was further refined by a second coder (J.W.). Individual POA data were categorized into these agreed-upon categories. Similarly, other benefits/changes in clinics as a result of participation in the DCC were also initially broadly categorized by the interviewer then refined by a second coder (J.W.) and, through an iterative process, concordance was reached by both reviewers.

The primary outcome was efficacy of the course as defined by reported full or partial implementation of POA. Secondary outcomes included any reported benefit in the clinic that could be directly related to attending the course, common barriers encountered in POA implementation, if not desiring to come to course impeded efficacy, and if not agreeing with group's POA impeded efficacy.

\section{2 | Quantitative design}

Initially, the course evaluation only consisted of daily subjective reviews of each speaker. While valuable knowledge about speakers was obtained, it did not provide information about course effectiveness, as defined by changing/improving diabetes care knowledge, attitudes, and behaviours. To address this, we developed a pre-course and post-course survey. In order to develop the survey, DCC organizers, including three physicians, two nurses (one of which is a CDE), and one $\mathrm{PhD} / \mathrm{MSW}$, developed questions to address knowledge, attitudes, and behaviours throughout the course by addressing specific topics each felt was important. An 18-question survey was developed that addressed at least one main topic from each didactic session. Questions were either multiple choice demographic/knowledge questions or six-point scale questions ( 1 = strongly disagree to $6=$ strongly agree). The same survey was administered on paper at the beginning of the course and at the end of the course, which included VTC participants. The initial pre- and post-survey were implemented in April 2016, which were amended (maintaining 18 questions) for the September 2016 course. The September 2016 course results from on location participants will be presented.

Primary outcomes were improved knowledge, skills, and intention to change clinical practice as pertains to comprehensive diabetes care and the material presented at the DCC. These do not specifically match with a traditional knowledge, attitudes, and behavior evaluation. However, we noted that we truly could not evaluate behaviours given our limited time from pre- to post-survey responses, rather only the intention to change behavior or clinical practice. We felt that attitude and intention to change behavior were similar constructs, so categorized both under intention to change clinical practice. Also, more pertinent to the hands-on module of the DCC, the category of skills was included, which could be considered a subset of knowledge but was independently evaluated in order to highlight an area of our course crucial to comprehensive diabetes care.

\section{3 | RESULTS}

\subsection{Qualitative results}

Out of 222 participants representing 77 clinics (if participants were from the same clinic but attended a different DCC session, this was counted as a separate clinic), 101 participants had an available POA, but only 87 participants had active email addresses (likely due to transient nature of military personnel). Disciplines represented by participants were dominated by nursing (50\%), which included RNs, LVNs, CDEs, Disease Managers, and Health Care Integrators; followed by 
providers (32\%), which included MDs, DOs, Residents, Fellows, NPs, and PAs; PharmDs (5\%); other (5\%), which included hospital leadership, dietitians, and technicians; and unknown (8\%) (Table 2).

The first round of emails was sent to all 87 participants that represented 46 unique clinics with 17 responses. The second round of emails was sent out to 46 participants, and eight additional responses were collected. Overall individual response rate (25/87) was $28.7 \%$ with 11 respondents attending the course on location (44\%) and 14 via VTC(56\%). As the overall response was relatively small, results were not separated into mode of attendance, on location versus VTC. Three clinics had two to three participants each that responded (full concordance noted within these clinics participant responses), there were a total of 19 clinics represented. This provided a $41 \%$ response rate (19/46) from individual clinics. Discipline of respondents were somewhat reflective of attendance with the vast majority being nurses (84\%) followed by providers (8\%) and other (8\%).

\section{2 | Outcomes}

The primary outcome of efficacy as defined by reported partial or full implementation of POAs was met by $12 / 19$ clinics (63\%). POAs varied in complexity; some were focused on one issue, while others were multifaceted. Therefore, one POA could have multiple categories. The categories, as defined and agreed upon through inter-rater reliability, were patient education (7); active monitoring (6), including immunizations, screening for diabetes, and intentional monitoring of patients with poorly managed blood sugar; staff education (4), including motivational interviewing, familiarization with CPG, and electronic medical record templates (Figure 1).

Twelve of 19 clinics (63\%) reported being able to implement part or all of their original POAs, and 17 of 19 clinics (89\%) reported improvements directly associated with attending the DCC. Benefits included improving multidisciplinary cooperation; routine screenings (labs, foot exams, ophthalmology); patient handouts and access to care; improving documentation; setting up a diabetes education class; increasing collaboration between the three military services; and even starting PI projects for other disease processes. Only two clinics did not report any improvement. In one of these clinics, the only participant was a disease manager that was relieved of duty 2 days after returning from the DCC. In the other clinic, the participating provider reported that she did not have any time to dedicate to the PI project and all other participants that attended the DCC were no longer employed at the clinic.

Individual clinics reported obstacles (often multiple) to implementation of POAs including staff turnover or loss (9), lack of time (8), under manning/overworked (8), provider pushback (7), lack of leadership support (6), knowledge deficits (5), system issues (5), underutilization of resources/team members (4), and communication (3) (Figure 2).

Other secondary outcomes had relatively small numbers. There were three participants that were "chosen" to go that did not specifically desire to come to the course. Only one of the three participants did not have full or partial implementation of POA in their clinic. The one that did not have implementation did see other benefits in her clinic (utilization of standardized patient resources used not only in her clinic, but other primary care clinics in the hospital). The majority of participants agreed with their group's POA; however, there were four participants that did not. Three of these four participants' clinics had partial or full implementation of POA, while the other one had other clinical improvements (the latter was the same individual that did not specifically want to come to the course that did not see partial or full POA implementation in her clinic).

\section{3 | Survey results}

For the September 2016 course, there were 104 participants, of which 57 attended on location. From those on location, we were able to obtain 53 pre-surveys and 45 post-surveys (Table 3). The VTC group included 47 participants, from which we were able to obtain 41 presurveys; however, only 13 completed the post-survey. Given this low response from the VTC participants, we will only present data

TABLE 2 Baseline participant roles for qualitative assessment

\begin{tabular}{|c|c|c|c|}
\hline & $\begin{array}{l}\text { Total Participants } \\
n=222(\%)\end{array}$ & $\begin{array}{l}\text { Potential Respondents } \\
n=87(\%)\end{array}$ & $\begin{array}{l}\text { Respondents } \\
n=25(\%)\end{array}$ \\
\hline Providers & $70(32)$ & $24(28)$ & $2(8)$ \\
\hline Independent MD/DO & 25 (11) & $9(10)$ & $1(4)$ \\
\hline Resident/fellow MD/DO & $22(10)$ & $7(8)$ & $0(0)$ \\
\hline Mid-level NP/PA & $23(10)$ & $8(9)$ & $1(4)$ \\
\hline Nurses & $112(50)$ & $52(60)$ & $21(84)$ \\
\hline RN/LVN & $75(34)$ & 34 (39) & $9(36)$ \\
\hline CDE & $5(2)$ & $2(2)$ & $1(4)$ \\
\hline Disease manager & $26(12)$ & $12(14)$ & $10(40)$ \\
\hline Health care integrator & $6(3)$ & $4(5)$ & $1(4)$ \\
\hline PharmD & $11(5)$ & $3(3)$ & $0(0)$ \\
\hline Other & $11(5)$ & $2(2)$ & $2(8)$ \\
\hline Hospital leadership & $3(1)$ & $1(1)$ & $1(4)$ \\
\hline Medical technician & $4(2)$ & $0(0)$ & $0(0)$ \\
\hline Dietitian & $4(2)$ & $1(1)$ & $1(4)$ \\
\hline Unknown & $18(8)$ & $6(7)$ & $0(0)$ \\
\hline
\end{tabular}

Note: Totals may not equal $100 \%$ due to rounding. 


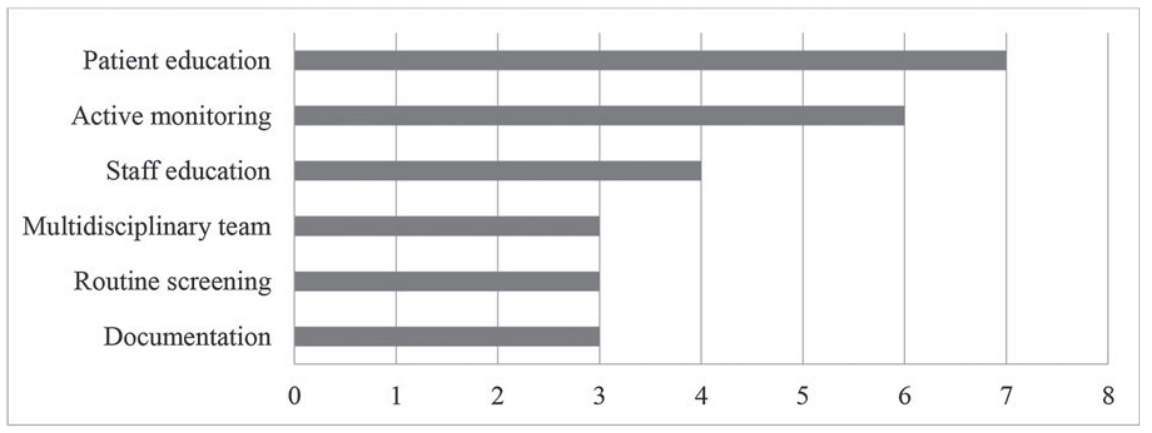

FIGURE 1 Plan of action categories (Total 26)

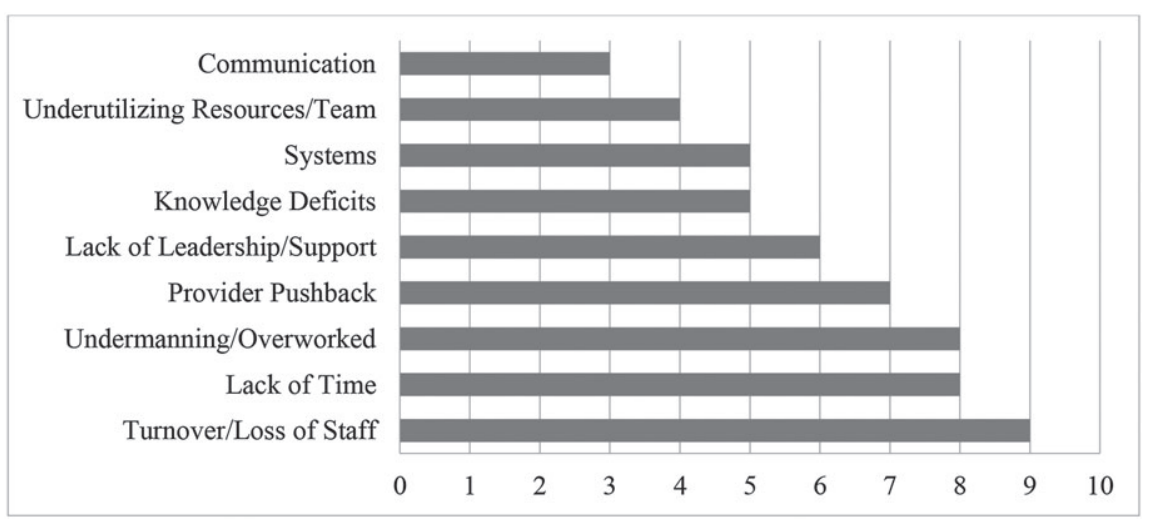

FIGURE 2 Reported plan of action obstacles

TABLE 3 Baseline participant roles for quantitative assessment

\begin{tabular}{lll} 
& \multicolumn{2}{l}{ Course Participants (on Location) } \\
\cline { 2 - 3 } & $\begin{array}{l}\text { Pre-test } \\
n=53(\%)\end{array}$ & $\begin{array}{l}\text { Post-test } \\
n=45(\%)\end{array}$ \\
\hline Providers & $18(34 \%)$ & $18(40 \%)$ \\
\hline Clinical nurse & $12(23 \%)$ & $10(22 \%)$ \\
\hline Disease manager & $7(13 \%)$ & $7(16 \%)$ \\
Pharm D & $4(8 \%)$ & $4(9 \%)$ \\
\hline Technician & $3(6 \%)$ & $3(7 \%)$ \\
Other & $9(17 \%)$ & $3(7 \%)$ \\
\hline
\end{tabular}

Note: Totals may not equal $100 \%$ due to rounding.

from those who attended in person. Self-reported roles for this iteration were reflective of previous courses, mainly nursing (42\%), followed by providers (40\%), and others (18\%) to include technician, dietitian, and PharmD.

Knowledge-based questions showed significant improvement in basic familiarity with insulin pumps $(P<0.01)$, knowledge of behaviours to prevent macrovascular complications $(P<0.01)$, and knowledge of cost-effective methods of utilizing self-monitored blood glucose levels $(P<0.01)$. The majority of providers $(97 \%)$ reported acquiring new knowledge about initiating and titrating insulin despite $89.5 \%$ feeling confident in their use of insulin prior to the course. Skills-based questions showed significant improvement in ability to demonstrate glucose meters to patients $(P<0.01)$ and ability to perform a comprehensive foot exam $(P=0.01)$. Intention to change clinical practice was demonstrated by $87.8 \%$ of participants who reported a need to revise current preoperative processes for patients with diabetes. In addition, participants' likelihood to use online resources presented at the DCC significantly increased $(P<0.01)$, and an enriched concept of team-based care was noted by an increase in perceived responsibility of technicians $(P<0.01)$, nurses $(P=0.01)$, and disease managers $(P=0.02)$ to ensure a foot exam was performed.

\section{4 | DISCUSSION}

We saw many improvements from our qualitative and quantitative PI assessments that may lead to improved health care delivery for patients with diabetes and CPG adherence. This does not assess hard outcomes like haemoglobin A1c (HgbA1c) reduction; however, we do not feel this would be the appropriate assessment tool. If a clinic's chosen POA was to improve foot exams on patients with diabetes with correlating documentation, this would not necessarily improve HgbA1c. This is why we felt reported partial or full POA implementation was a better marker of efficacy that would be directly related to CPG adherence, which had an overall 63\% efficacy as regards partial or full implementation of POAs. In addition, $89 \%$ reported improvements directly associated with attending the DCC. We hypothesize that as continued PI becomes a focus in these clinics, higher adherence to CPGs would take hold with eventual benefit in these hard outcomes. This idea was seen in a 2016 study that used onsite practice facilitation, usually trained nurses, to teach PI in clinics that led to an absolute improvement of $19 \%$ in the proportion of patients achieving $\mathrm{HgbA} 1 \mathrm{c}<7 \%$ and a significant reduction in the number of patients with $\mathrm{HgbA} 1 \mathrm{c}>9 \%{ }^{10}$

Similar to many other evaluations of CPD, we were able to show that by attending our course on location there was a significant shortterm gain in participants' knowledge. Even when participants felt knowledgeable on a topic, such as provider confidence with insulin 
initiation and titration, the DCC provided new information for clinical use. This was demonstrated by $97 \%$ of providers acquiring new knowledge in this area despite $89.5 \%$ feeling confident before the course. This was similarly seen in regards to $87.8 \%$ of participants indicating feeling like their current preoperative process for patients with diabetes was in need of revision. The DCC is multifaceted as its interactive component also helps to increase skills like demonstration of glucose meters and performing a comprehensive foot exam on patients with diabetes.

In review of the literature, interprofessional CPD courses will help to improve patient safety and deliver high-quality health care through team building and role clarification. ${ }^{4,6}$ We feel that all team members need to be familiar with the CPGs in order to effectively apply the multifaceted care recommended for patients with diabetes. Our course was designed for primary care teams (provider, nurse, technician, PharmD, etc.) to attend; however, often only partial teams or solo team members attend. While this may not be optimal, it is the realworld application and still proved efficacious.

Our current attendance is dominated by the nursing field (50\%), albeit they are in different practical roles such as clinical nurse, disease manager, health care integrator, and CDE. This is followed by provider (32\%); PharmD (5\%); and hospital leadership, medical technician, and dietitian ( $<5 \%$ each). To improve CPG treatment adherence, we feel it is important for provider attendance; however, for PI implementation, perhaps it is more important for nursing attendance. This may explain the overall efficacy of the course, based on reported partial or full implementation of POA. While we feel full team attendance is important, especially to facilitate a team approach to diabetes care, optimal attendance by participant role is not known. This would be an area of further investigation for best post-course efficacy.

Participant selection should be reviewed as well. We would like to see motivated individuals that want to come to the course as this may lead to higher likelihood of PI completion and even continuation. However, we demonstrated that even if participants did not want to participate, there was still partial or full POA implementation or at least some clinical benefit (three out of three). Ideally, we would want participants to at least be continuing their current role in their current clinic (or be retained in a clinic that provides diabetes care) for at least 1 year after attendance. Also, we would like to see participants be able to have time allocated to $\mathrm{PI}$ in the clinic. This may not mean that everyone on the team needs to have dedicated time, but at least one person on that team should have time allocated to the PI process.

We found the main obstacles to be staff turnover or loss, lack of time, under manning/overworked, provider pushback, lack of leadership/support, knowledge deficits, and system issues. The literature describes that the common obstacles to clinical improvement similarly include lack of PI skills and leadership support, clinical inertia, complex recommendations difficult to implement, and resistance by patients and families. ${ }^{11} \mathrm{~A}$ recent special report from the American College of Cardiology/American Heart Association task force on CPGs indicates similar obstacles include time, human resources, scepticism, lack of knowledge of guidelines, and older age. ${ }^{3}$ Our most commonly reported obstacle was staff turnover or loss. While this is not military specific, the military is known for predictably high turnover in clinical care. The driving force behind PI projects is often physician or nurse led. Providers and nurses are often not with a clinic for more than 3 to 4 years. Often gaps in filling the position leave understaffed providers and nurses covering their responsibilities. Ancillary duties are often the first to get dropped, like PI projects, especially if early in evolution. Also, while medical technicians may be at a specific Military Treatment Facilities for 3 to 4 years, they are often moved from clinic to clinic multiple times necessitating extra time for training new staff and disrupting continuity of care.

There are several important limitations. First, this was a PI project and was retrospective in nature. Also, while the majority of attendees were nurses, an even larger portion of respondees were nurses which could introduce unexpected bias. In regard to the qualitative aspect, there was high reported partial or full implementation of POAs and/or clinical improvement after attending the DCC, but this was selfreported. Also, despite a respectable response rate (28.7\%) from valid potential respondents (POA turned in with valid email address), overall response rate from total participants (222) was only $11.3 \%$, which may not truly represent the participants as a whole. We likewise do not have outcomes of individual POAs and whether they actually improved CPG adherence. In regard to the quantitative aspect, due to poor responses and tracking for VTC participants, our current evaluation of the course did not include the VTC participants and can only be generalized to on location participants. Finally, while we demonstrate improved shortterm knowledge and confidence in certain skills related to diabetes care this does not necessarily translate into actual practice.

\section{5 | CONCLUSION}

The DCC is an innovative, team-based, interprofessional, didactic, and interactive, 3-day comprehensive diabetes course aimed at primary care teams in the military health system. This short CPD course is focused around developing a PI project based on local CPG adherence deficiencies while providing comprehensive diabetes care knowledge. Our initial data collected from our own PI project (or POA) on the course has shown the course to be efficacious in reported PI implementation. Our hope is that the PI project taken back from the course is just the first of many to continue improvement of diabetes CPG adherence. We also have shown that on location attendance at the DCC improved short-term knowledge, awareness of diabetes resources, and confidence in diabetes-care related clinical skills while fostering a mentality of team-based responsibility for CPG adherence. Future research should be directed at clinical CPG adherence and whether there was continued $\mathrm{PI}$ and if this relation leads to improved patient outcomes like HgbA1c reduction.

\section{ORCID}

Darrick Beckman (D) http://orcid.org/0000-0003-0739-5618

Jana Wardian (D) http://orcid.org/0000-0003-3025-686X

Mark W. True (I) http://orcid.org/0000-0003-3488-4193

\section{REFERENCES}

1. Vigersky RA, Fish L, Hogan P, et al. The clinical endocrinology workforce: current status and future projections of supply and demand. J Clin Endocrinol Metab. 2014;99(9):3112-3121. https://doi.org/ 10.1210/jc.2014-2257 
2. McMaughan DK, Huber JC Jr, Forjuoh SN, et al. Physician recommendation of diabetes clinical protocols. Hosp Top. 2016;94(1):15-21. https://doi.org/10.1080/00185868.2016.1142313

3. Chan WV, Pearson TA, Bennett GC, et al. ACC/AHA special report: clinical practice guideline implementation strategies: a summary of systematic reviews by the NHLBI implementation science work group: A Report of the American College of Cardiology/American Heart Association Task Force on Clinical Practice Guidelines. J Am Coll Cardiol. 2017;69(8):1076-1092. https://doi.org/10.1016/j.jacc.2016.11.004

4. Sachdeva AK. Continuing professional development in the twenty-first century. J Contin Educ Health Prof. 2016;36(Suppl 1):S8-S13. https:// doi.org/10.1097/CEH.0000000000000107

5. Forsetlund L, Bjørndal A, Rashidian A, et al. Continuing education meetings and workshops: effects on professional practice and health care outcomes. Cochrane Database Syst Rev. 2009;(2):CD003030. https://doi.org/10.1002/14651858.CD003030.pub2

6. Yu CH, Halapy H, Kaplan D, Brydges R, Hall S, Wong R. Effects of a longitudinal Interprofessional educational outreach program on collaboration. J Contin Educ Health Prof. 2016;36(1):24-31. https://doi.org/ 10.1097/CEH.0000000000000020

7. Cady RF. "Becoming a high reliability organization-operational advice for hospital leaders" report. JONAS Healthc Law Ethics Regul. 2008;10(2):33. https://doi.org/10.1097/01.NHL.0000300780.65358.e0
8. Chassin MR, Loeb JM. High-reliability health care: getting there from here. Milbank Q. 2013;91(3):459-490. https://doi.org/10.1111/14680009.12023

9. Matthews V, Schierhout G, McBroom J, et al. Duration of participation in continuous quality improvement: a key factor explaining improved delivery of type 2 diabetes services. BMC Health Serv Res. 2014;14(1):578. https://doi.org/10.1186/s12913-014-0578-1

10. Cykert S, Lefebvre A, Bacon T, Newton W. Meaningful use in chronic care: improved diabetes outcomes using a primary care extension center model. N C Med J. 2016;77(6):378-383.

11. Vander Schaaf EB, Seashore CJ, Randolph GD. Translating clinical guidelines into practice: challenges and opportunities in a dynamic health care environment. N C Med J. 2015;76(4):230-234. https:// doi.org/10.18043/ncm.76.4.230

How to cite this article: Beckman D, Wardian J, Sauerwein TJ, True MW. Evaluation of an interprofessional continuing professional development course on comprehensive diabetes care: $A$ mixed-methods approach. J Eval Clin Pract. 2019;25:148-154. https://doi.org/10.1111/jep.13033 